\title{
Histological and immunohistochemical study on human placental tissue in normal pregnancy and preeclampsia
}

\author{
Noha Abdellatif Ibrahim ${ }^{1}$, Doaa Mabrouk Khaled ${ }^{2}$ \\ ${ }^{1}$ Histology Department, Faculty of Medicine, Fayoum University, Cairo, Egypt \\ ${ }^{2}$ Histology Department, Faculty of Medicine, MUST University, Cairo, Egypt \\ Email address: \\ nh_ebrahim@yahoo.com (N. A. Ibrahim), nohaabdellatif60@gmail.com (D. M. Khaled)
}

\section{To cite this article:}

Noha Abdellatif Ibrahim, Doaa Mabrouk Khaled. Histological and Immunohistochemical Study on Human Placental Tissue in Normal Pregnancy and Preeclampsia. Cell Biology. Vol. 2, No. 6, 2014, pp. 72-80. doi: 10.11648/j.cb.20140206.13

\begin{abstract}
Background: Preeclampsia is a serious disorder affecting the mother. It may be responsible for more than 50,000 maternal deaths worldwide each year. It is a major killer of both mother and fetus. Aim: To demonstrate the morphological changes of human placental tissue in pre-eclamptic pregnancies compared with the normal by using histological and immunohistochemical techniques. Materials \& Methods: Twenty pregnant women were included in this study. 10 of them were diagnosed as having moderate pre-eclampsia and considered as the pre-eclamptic (PE) group, while 10 women were clinically normal and considered as the control group. Placental specimens were obtained after either vaginal delivery or caesarian section and processed for H\&E and Masson's trichrome stains, as well as for immunohistochemical stains for eNOS and HIF$1 \alpha$. Morphometric measurement of number of syncytial knots, area $\%$ of collagen fibers, eNOS and HIF-1 1 .were done followed by statistical analysis. Results: PE group revealed thickened wall of fetal blood capillaries, crowded degenerating villi with decreased intervillous spaces, intravillous and perivillous fibrinoid deposition. Numerous syncytial knots and exfoliated trophoblast cells were also observed. There was thickened layer of subchorionic fibrinoid. Increased area\% of collagen fibers, HIF-1 $\alpha$. immunoreactivity and decreased area $\%$ of eNOS immunoexpression were found. Conclusions: As evidenced by the previous results, this study concluded various placental changes detected by histological and histochemical techniques. The findings have clarified a significant correlation between eNOS and HIF- $1 \alpha$ immunoexpression and pre-eclampsia. Whether this relation is causal or effect relationship, this may need further investigations for better management and avoidance of consequences of pre-eclampsia.
\end{abstract}

Keywords: Pre-Eclampsia, eNOS, HIF-1 $\alpha$, Histological, Immunohistochemical

\section{Introduction}

Placenta is a unique organ that arises de novo. It is essential for the growth and development of the fetus in the uterus. Despite its central role in pregnancy, placenta so far is a poorly understood organ. Being an organ of paramount importance, examination of placenta is necessary for both mother and infant. It can be informative for later management of both infant and the mother $(1,2)$.

Preeclampsia is a serious disorder affecting the mother. Its incidence is about 5-10\% among pregnancies. Pre-eclampsia may be responsible for more than 50,000 maternal deaths worldwide each year. It is a major killer of both mother and fetus. Pre-eclampsia is diagnosed by blood pressure elevation and proteinuria. It develops after 20 weeks' of gestation. Earlier observations have revealed resolving of all signs and symptoms of this disease after delivery of placenta. Therefore, placenta may be a source of this disease $(3,4,5)$.

The pathophysiology of preeclampsia involves both maternal and fetal/placental factors. Abnormalities in the development of placenta early in pregnancy may lead to relative placental underperfusion/hypoxia/ischemia. This subsequently leads to release of antiangiogenic factors into the maternal circulation with alteration of maternal systemic endothelial function. This causes hypertension and other manifestations of the disease. Despite intensive researches the exact cause of disorder is still unknown (6).

L-arginine-nitric oxide pathway could also play a central role in both normal pregnancy and in preeclampsia. Many elements of the 1-arginine-NO signaling pathway may be abnormal, including reduced endothelial NO synthase (eNOS) enzyme activity. Nitric oxide (NO) is a potent vasodilator synthesized by the endothelial NO synthase (eNOS) in 
endothelium of muscularized fetoplacental vessels. NO release has shown to maintain low vascular resistance in the fetoplacental circulation in humans in vitro. This may suggest an important role of eNOS in systemic cardiovascular changes in pregnancy (7).

Hypoxia-inducible factor- $1 \alpha(\mathrm{HIF}-1 \alpha)$ is one of the factors which is important for placental development. It is a transcription factor that is transiently expressed as a protein. It can also bind to hypoxia-response elements (HREs) in the promoters of several genes involved in the adaptation to an environment of hypoxia (8).

The aim of this work was to demonstrate the morphological changes of human placental tissue in preeclamptic pregnancies compared with the normal by using histological and immunohistochemical techniques.

\section{Materials and Methods}

\subsection{Patients}

This study was a comparative study. It was conducted at the Faculty of Medicine, MUST University Hospital. Written informed consent was obtained from all participants before their participation.

\subsection{Selection of Cases}

Twenty pregnant women from the Obstetrics and Gynaecology delivery suite, MUST University hospital were included in this study. 10 of them were included in the moderate pre-eclampsia group, while 10 women were clinically normal and considered as the control group. All participants fulfilled the inclusion and exclusion criteria.

The inclusion criteria included:

- Age from 20 to 35 years.

- Primigravida.

- Gestational age between 28 to 38 weeks.

- Willingness to participate and signing consent form after explanation of the aim of the study.

The exclusion criteria included:

- Preexisting diabetes mellitus

- History of maternal diseases such as chronic renal disorders, essential hypertension, autoimmune disease, or thrombophilic conditions.

- Evidence of fetal anomalies or hydrops fetalis.

- Gestation diabetes or any medications.

\subsection{Divisions of the Groups}

Group I (control group): included ten healthy primigravidae, who were normotensive, normoglycemic, non obese, with no abnormality on routine clinical examination. They were admitted to the delivery unit for either vaginal or cesarian delivery in the third trimester.

Group II: (PE group): included ten patients selected on the basis of criteria of moderate PE of the National Institute for Health and Care Excellence (NICE, UK). These criteria were: $\mathrm{BP}$ is 150 to $159 \mathrm{mmHg}$ systolic and/ or 100 to $109 \mathrm{mmHg}$ diastolic on 2 occasions at least 6 hours apart, proteinuria (defined as urinary excretion of $>0.3 \mathrm{~g}$ protein in 24 hours).

\subsection{Sample Collection}

After delivery of the placentae, they were washed to remove any clotted blood. All placental samples were of similar thickness, Two sections each from central and peripheral areas were taken. Additional sections were taken from grossly abnormal lesions. Specimens $(2 \mathrm{~cm})$ were fixed in $10 \%$ neutral - buffered formalin. They were processed for paraffin sections. Five $\mu \mathrm{m}$ sections were cut and stained with H\&E and Masson's trichrome stains. Immunohistochemical staining was carried for the detection of expression of eNOS (Labvision, Thermoscientific, USA) rabbit polyclonal antibody, code no. PA1-37625. The reaction is membranous and cytoplasmic. The +ve control was the placenta. HIF1- $\alpha$ (Abcam, USA) mouse monoclonal antibody, code no. ab113642. The reaction is nuclear and cytoplasmic. The +ve control was the brain cancer tissue. An additional slide of placental specimen was treated with buffer solution instead of the same concentration of primary antibody in every run as the negative control.

\subsection{Morphometric Study}

The data were obtained using the image analyzer computer system (Leica Qwin 500, Leica, England) at Histology Department - Faculty of Medicine, Cairo University Kasr ElAini. The number of the syncytial knots was measured in 10 low power non-overlapping fields using binary mode. The area $\%$ of collagen fibers was measured in 10 low power non-overlapping fields. While, the area $\%$ of eNOS and HIF$1 \alpha$ immunoreaction were measured in 10 high-power nonoverlapping fields using binary mode.

\subsection{Statistical Analysis}

Data were expressed as group mean \pm SD. The statistical analysis was carried out using student t-test, with SPSS version 10 (SPSS, Chicago, Illinois, USA). All results were compared one by one with the results of the control group. A P value of 0.05 or less was considered as statistically significant.

\section{Results}

\subsection{Histological Results}

\subsubsection{Hematoxylin and Eosin}

The control group (group I) showed chorionic villi covered by syncytiotrophoblasts and separated by intervillous spaces containing maternal blood. Each villous had a core of stroma containing fetal blood capillaries and stromal cells. Syncytial knots, syncytial sprouts and syncytial strands were noticed. Exfoliated trophoblast cells in the intervillous spaces were observed (Fig. 1). A compact layer of chorionic plate covered by Langhan's layer of fibrinoid, which in turn was covered by syncytiotrophoblasts towards the intervillous spaces were revealed. Stem villus fetal blood capillaries were also noticed (Fig. 2). 
The PE group (group II) showed thickened wall of fetal blood capillaries, crowded degenerating villi with decreased intervillous spaces, intravillous and perivillous fibrinoid deposition. Numerous syncytial knots and exfoliated trophoblast cells were observed. Ruptured villi with extravasation of fetal RBCs into the maternal space were revealed (Figs. 3, 4, 5). Multi-layered structure of chorionic plate, consisting of amniotic epithelium, amniotic mesenchyme and spongy layer, followed by a compact layer of chorionic mesenchyme were demonstrated. Chorionic plates were covered by thickened Langhan's layer of fibrinoid, with loss of syncytiotrophoblast layer towards the intervillous spaces. Hemorrhage in the intervillous spaces and apparently decreased fetal blood capillaries were noticed (Fig. 6).

\subsubsection{Masson's Trichrome Stain}

The control group showed delicate collagen fibers within villous stroma and around fetal blood capillaries (Fig. 7). While the PE group demonstrated dense collagen fibers within villous stroma and around fetal blood capillaries (Fig. 8).

\subsection{Immunohistochemical Results}

\subsection{1. eNOS Immunoreactivity}

In the control group, strong positive cytoplasmic immunoreaction in syncytiotrophoblasts and endothelial lining of fetal blood capillaries was revealed (Figs. 9a, b). While the PE group showed negative cytoplasmic immunoreaction of most syncytiotrophoblasts and positive cytoplasmic immunoreaction in few cells (Figs. 10a, b).

\subsubsection{HIF-1a Immunoreactivity}

In the control group, negative nuclear and cytoplasmic immunoreaction was observed (Figs. 11a, b). In the PE group, strong positive nuclear and cytoplasmic immunoreaction in syncytiotrophoblasts was revealed (Figs. 12a, b).

\subsection{Morphometric Results}

\subsubsection{Number of Synsytial Knots}

There was a significant increase $(\mathrm{P} \leq 0.05)$ in the mean number of syncytial knots in the PE group compared with the control group (Table).

\subsubsection{Area \% of Collagen Fibers}

A significant increase $(\mathrm{P} \leq 0.05)$ in the mean area $\%$ of collagen fibers was found in the PE group compared with the control group (Table).

\subsubsection{Area \% of eNOS}

There was a significant decrease $(\mathrm{P} \leq 0.05)$ in the mean area $\%$ of eNOS immunoreactivity in the pre-eclamptic group compared with the control group (Table).

\subsubsection{Area \% of HIF-1 $\alpha$}

There was a significant increase $(\mathrm{P} \leq 0.05)$ in the mean area $\%$ of HIF-1 $\alpha$ immunoexpression in the PE group compared with the control group (Table).

Table 1. Mean number of syncytial knots, Mean area \% of collagen fibers, eNOS immunoreaction and HIF-1 $\alpha$ immunoreaction.

\begin{tabular}{llll}
\hline \multirow{2}{*}{ Groups } & $\begin{array}{l}\text { Mean } \pm \text { SD number of } \\
\text { syncyteal knots }\end{array}$ & $\begin{array}{l}\text { Mean } \pm \text { SD area \% of collagen } \\
\text { fibers }\end{array}$ & Mean \pm SD area \% of eNOS \\
\hline Control & $5 \pm .02$ & $8.5 \pm 1.86$ & $22.1 \pm 2.1$ \\
PE & $12 \pm .1^{*}$ & $31 \pm 3.25^{*}$ & $4.3 \pm .8^{*}$ \\
\hline
\end{tabular}

$* \mathrm{P} \leq 0.05$, significant difference compared with control

\subsection{Legends}

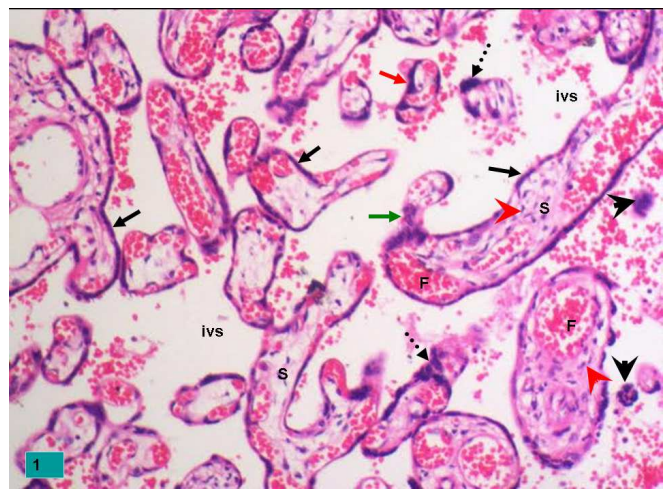

$\mathrm{H} \& \mathrm{E}, \mathrm{X} 200$

Fig. 1. A photomicrograph of a section in the human placenta of a control group (group I) showing chorionic villi covered by syncytiotrophoblasts (thin arrows) and separated by intervillous spaces (ivs) which contain maternal blood. Villous stroma $(S)$ containing fetal blood capillaries $(F)$ and stromal cells (red arrow heads) are also shown. Syncytial knots (dotted arrows), syncytial sprout (red arrow) and syncytial strand (green arrow) are noticed. Exfoliated trophoblast cells (black arrow heads) in the intervillous spaces are observed.

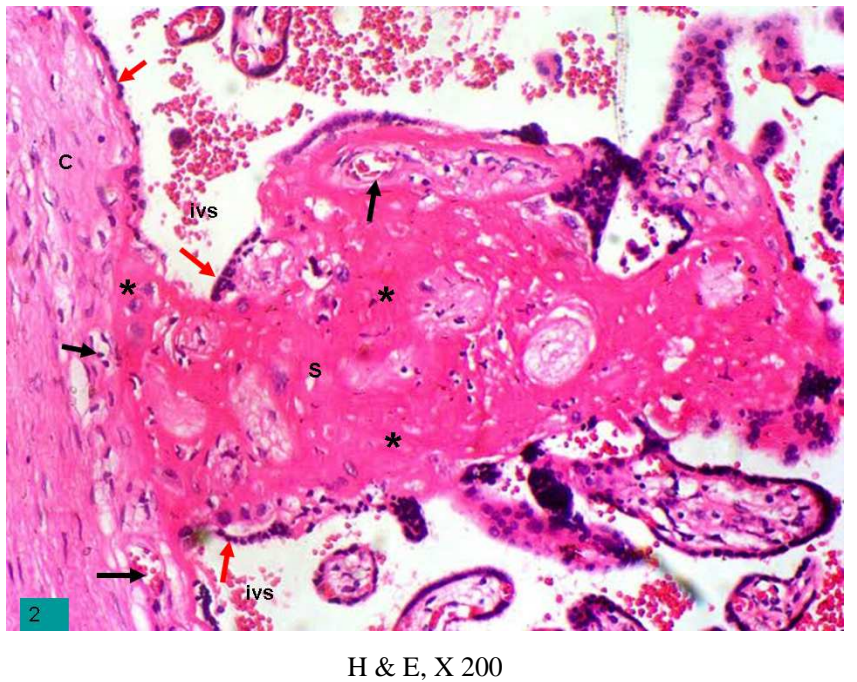

Fig. 2. A photomicrograph of a section in the human placenta of a control group (group I) showing a compact layer of chorionic plate $(C)$. Chorionic plates are covered by Langhan's layer of fibrinoid (*), which in turn is covered by syncytiotrophoblast (red arrows) towards the intervillous spaces (ivs). Notice the stem villus (S) and fetal blood capillaries (black arrows). 


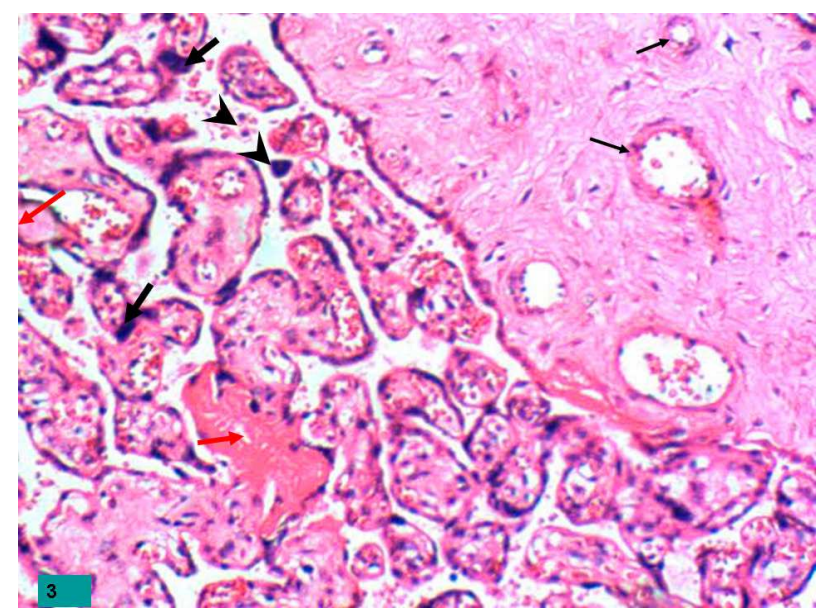

$H \& \mathrm{E}, \mathrm{X} 200$

Fig. 3. A photomicrograph of a section in the human placenta of a PE group (group II) showing thickened fetal blood capillaries (thin arrows), crowded degenerating villi with decreased intervillous spaces, intravillous fibrinoid deposition (red arrows). Syncytial knots (thick arrows) and exfoliated trophoblast cells (arrow heads) are observed.

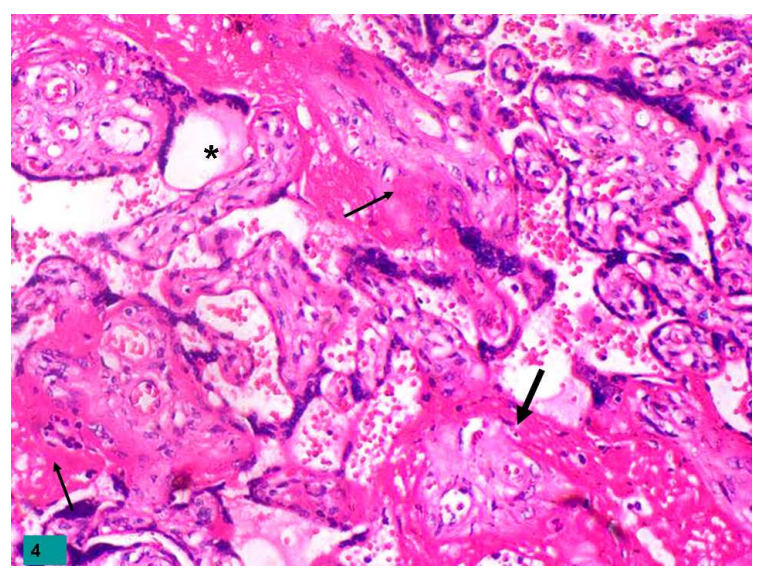

$H \& \mathrm{E}, \mathrm{X} 200$

Fig. 4. A photomicrograph of a section in the human placenta of a PE group (group II) showing crowded villi with decreased intervillous spaces, intravillous (thin arrows) and perivillous (thick arrows) fibrinoid deposition. Notice a necrotic villus (*).

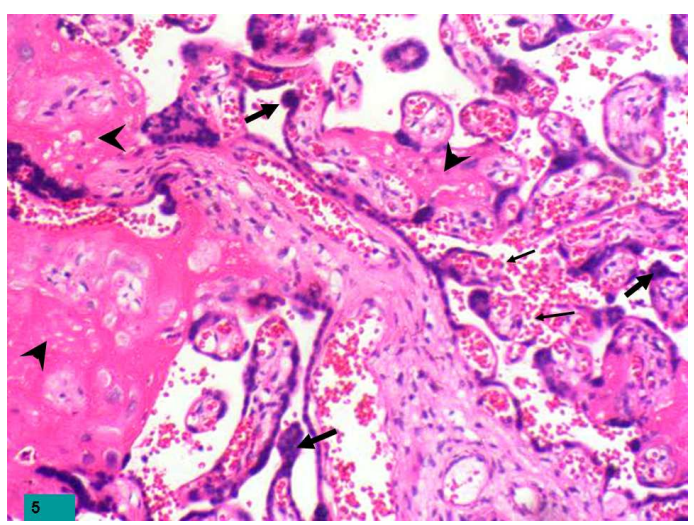

$\mathrm{H} \& \mathrm{E}, \mathrm{X} 200$

Fig. 5. A photomicrograph of a section in the human placenta of a PE group (group II) showing numerous syncytial knots (thick arrows), intravillous fibrinoid degeneration (arrow heads) and ruptured villi (thin arrows) with extravasation of fetal RBCs into the maternal space.

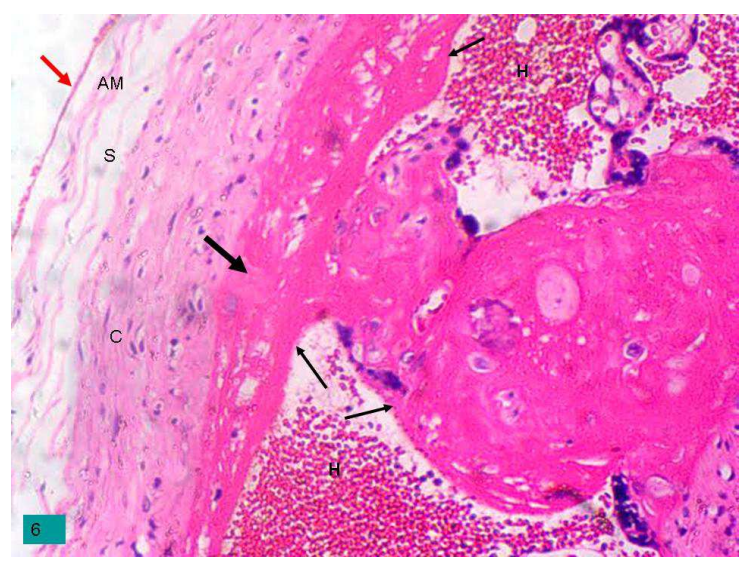

$H \& \mathrm{E}, \mathrm{X} 200$

Fig. 6. A photomicrograph of a section in the human placenta of a PE group (group II) showing multi-layered structure of chorionic plate, consisting of amniotic epithelium (red arrow), amniotic mesenchyme (AM), spongy layer $(S)$, followed by a compact layer of chorionic mesenchyme $(C)$. Chorionic plates are covered by thickened Langhan's layer of fibrinoid (thick arrow), with loss of syncytiotrophoblast layer (thin arrows) towards the intervillous spaces. Notice the hemorrhage $(H)$ in the intervillous spaces and apparently decreased fetal blood capillaries.

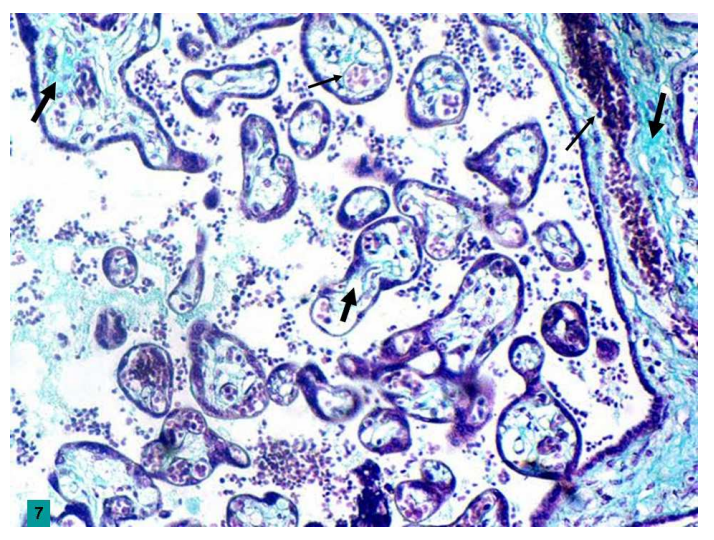

MT stain, X 200

Fig. 7. A photomicrograph of a section in the human placenta of a control group (group I) showing delicate collagen fibers within villous stroma (thick arrows) and around fetal blood capillaries (thin arrow).

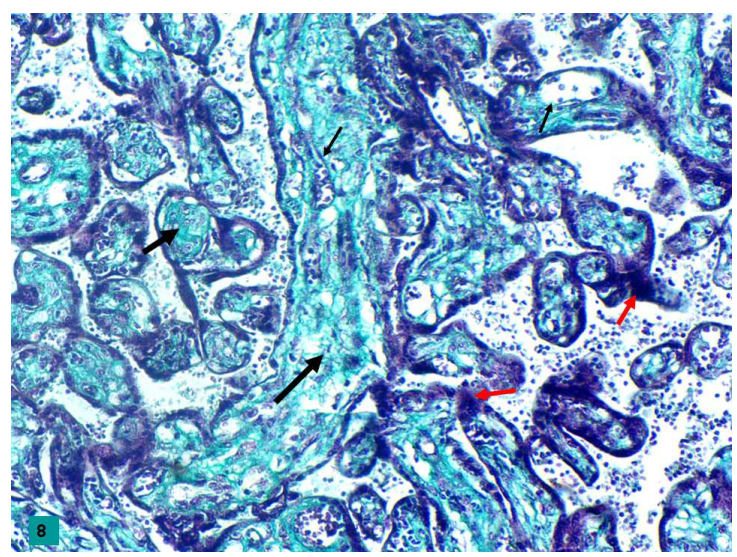

MT stain, X 200

Fig. 8. A photomicrograph of a section in the human placenta of a PE group (group II) showing dense collagen fibers within villous stroma (thick arrow) and around fetal blood capillaries (thin arrow). Note he large number of sycyteal knots (red arrows) and the crowdness of villi. 


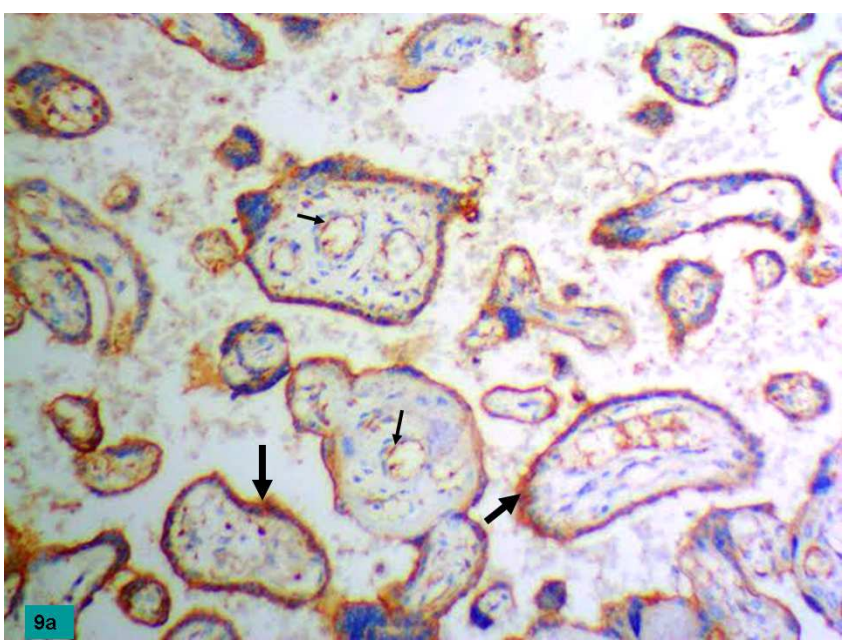

(a) eNOS immunostain, X 200

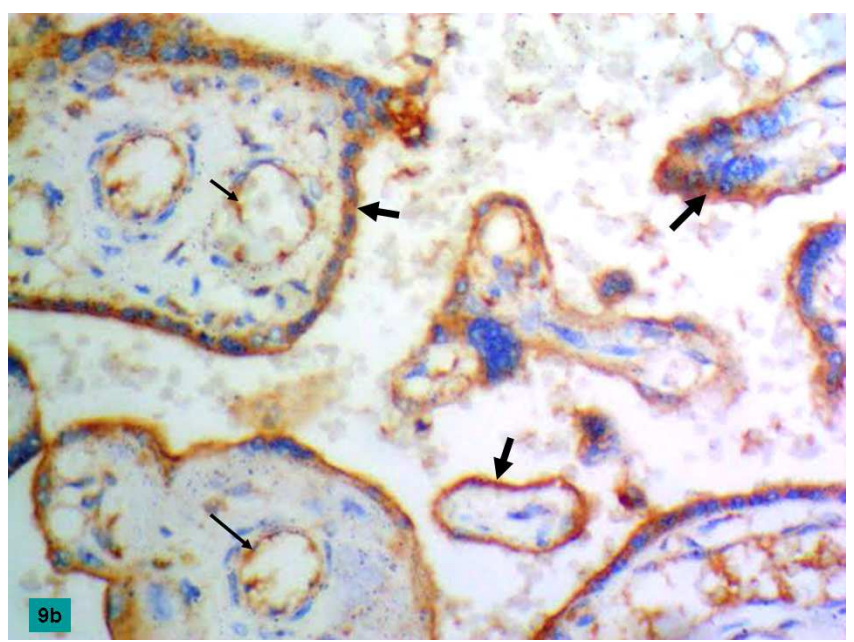

(b) eNOS immunostain, $\mathrm{X} 400$

Figs. 9a, b. photomicrographs of a section the human placenta of a control group (group I) showing a: strong positive cytoplasmic immunoreaction of eNOS in syncytiotrophoblasts (thick arrows) and endothelial lining of fetal blood capillaries (thin arrows). b: a higher magnification of the previous photomicrograph.

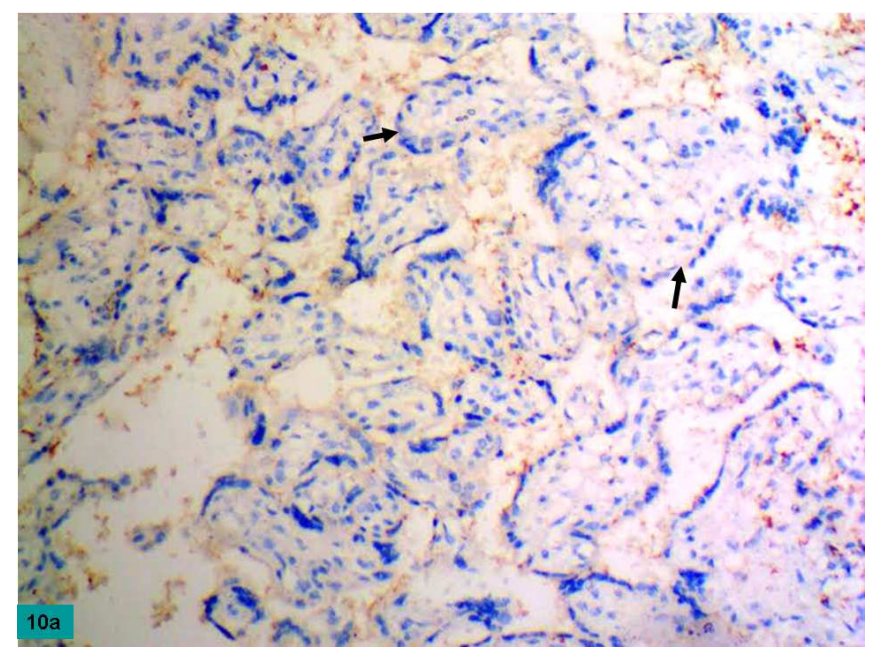

(a) eNOS immunostain, X 200

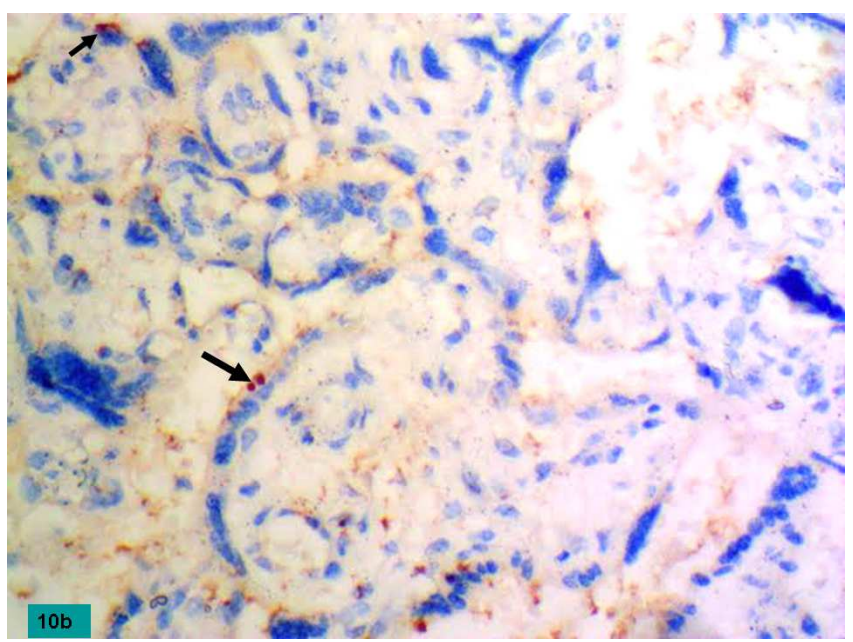

(b) eNOS immunostain, X 400

Figs. 10a, b. photomicrographs of a section the human placenta of a PE group (group II) showing (a) Negative cytoplasmic immunoreaction of eNOS (arrows). (b) Positive cytoplasmic immunoreaction of eNOS in a few cells of syncytiotrophoblasts (arrows).

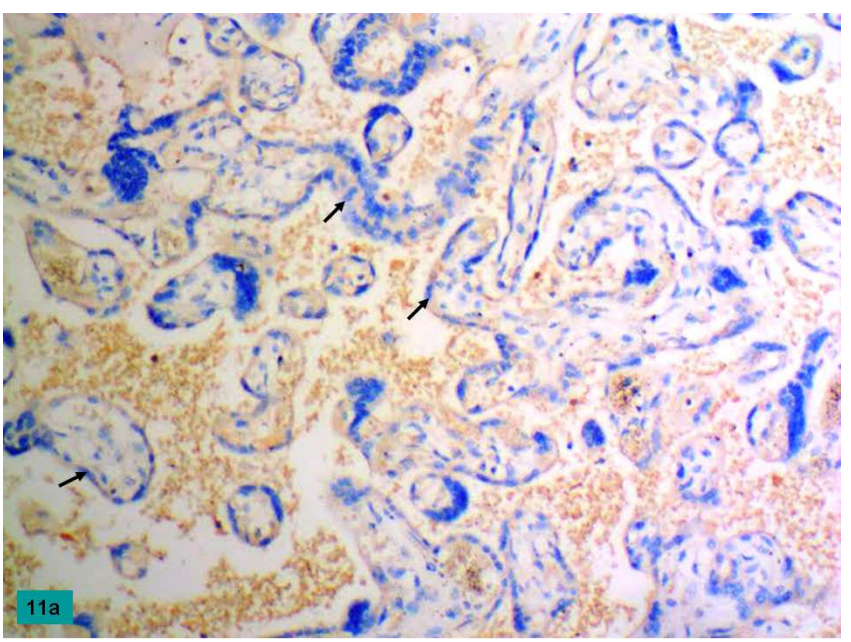

(a): HIF-1 $\alpha$ immunostain, X 200

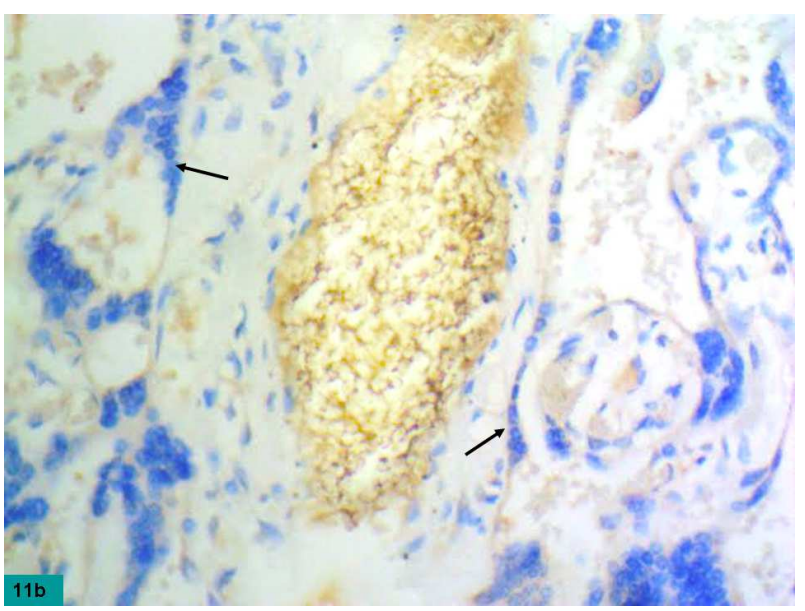

(b): HIF-1 $\alpha$ immunostain, X 400

Figs. 11a, b. photomicrographs of a section the human placenta of a control group (group I) showing a: negative nuclear and cytoplasmic HIF-1a immunoreaction in syncytiotrophoblasts (arrows). $b$ : a higher magnification of the previous photomicrograph. 


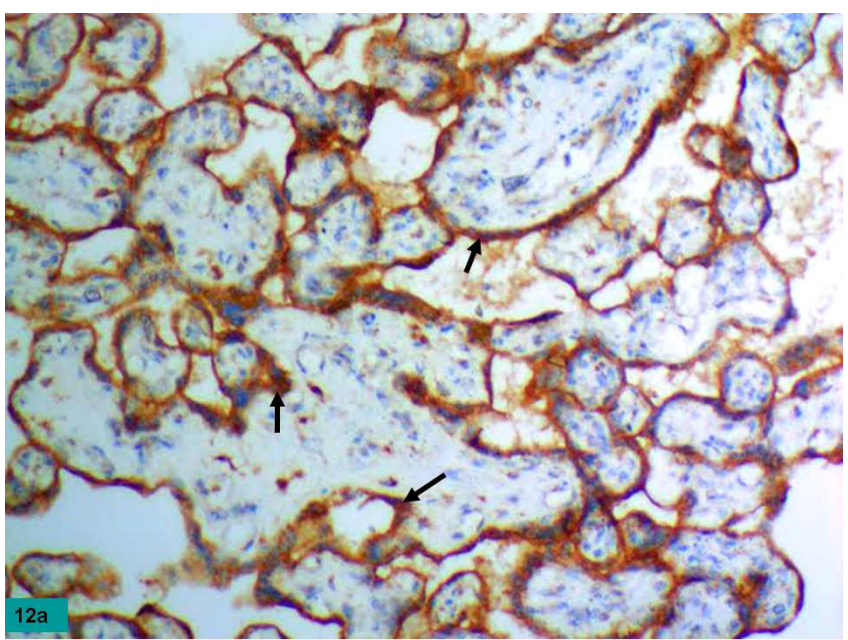

(a): HIF-1 $\alpha$ immunostain, X 200

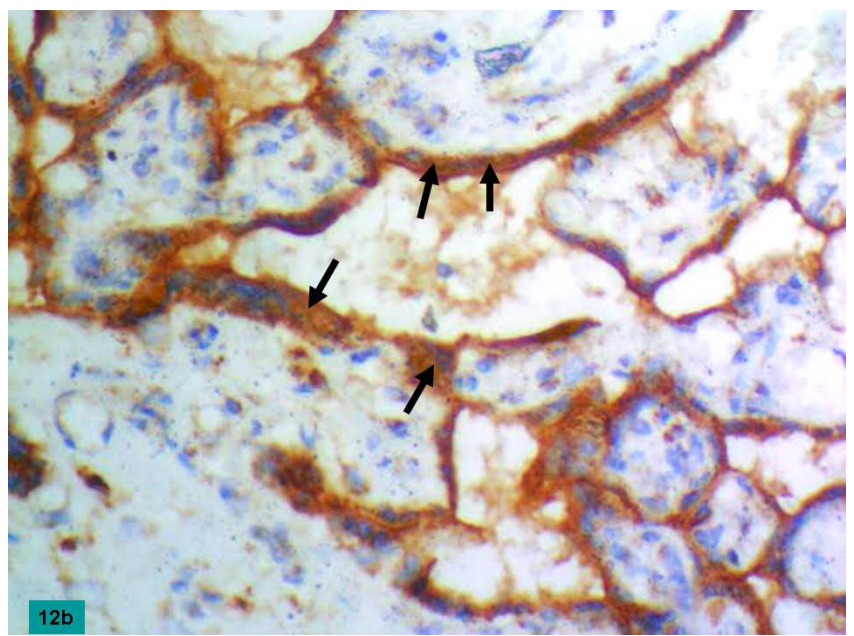

(b): HIF-1 $\alpha$ immunostain, X 400

Figs. 12a, b. photomicrographs of a section the human placenta of a PE group (group II) showing a: strong positive nuclear and cytoplasmic HIF-1a immunoreaction in syncytiotrophoblasts (arrows). Very crowded villi with decreased intervillous spaces are observed. $b$ : a higher magnification of the previous photomicrograph.

\section{Discussion}

In the present study, marked placental changes in the preeclamptic group were revealed. These changes were confirmed by histological, immunohistochemical and morphometric results.

Examination of the placenta is extremely important in attempting to understand the pathophysiology of preeclampsia. Only with careful gross, histological evaluation, along with clinical pathologic correlation, the underlying causes and recurrence risks could be understood. There is a great debate going on whether placental inefficiency is a primary cause, contributory factor or a result of preeclampsia.

In the present study, examination of pre-eclamptic placentae revealed thickened wall of fetal blood capillaries, crowded degenerating chorionic villi with decreased intervillous spaces, intravillous and perivillous fibrinoid deposition. Ruptured villi with extravasation of fetal RBCs into the maternal space were also revealed. Subchorionic fibrin deposition with decreased number of fetal blood capillaries in the chorionic plate, loss of syncytiotrophoblast shell and hemorrhage in the intervillous spaces were demonstrated. These results were in agreement with those of some authors $(1,2,9)$ who reported the same findings.

The fibrinoid deposits are structurally and chemically related to fibrin. It is formed by the activation of fibrinogen in the blood vessels. The fibrinoid deposits are present in all normal placentae. They increase in thickness during the pregnancy without affecting its function. The functional importance of the fibrinoid are quite complex. Besides their sealing effects, they also have a role in the immunologic "barrier" between feto-maternal tissue as well in the anchoring of the placenta (10). Increased fibrinoid formation may be related to micro-lesions of the syncytiotrophoblast. So, fibrinoid could envelope all necrotic material that results from placental degeneration. As these injuries make mesenchyma comes into contact with maternal blood. This leads to altered function of haemostatic factors interacting with activated protein $\mathrm{C}$ in the placenta. Where previous studies have shown increased activated protein $\mathrm{C}$ resistance leading to ineffective anticoagulant response (11). Deposition of fibrinoid prevents normal gas and nutritive exchange between the maternal and fetal circulations. This consequently can lead to fetal growth restriction (12).

Exfoliated trophoblasts in the maternal space and a significant increase in number of syncytial knots compared with the control group were also revealed. This was consistent with a previous study that revealed increased number of syncytial knots in preeclamptic placentae (13).

Syncytiotrophoblastic knots or syncytial knots are aggregates of syncytial nuclei at the surface of terminal villi. Syncytial knots are normally present, increasing in number with increasing gestational age. So, it can be used to evaluate villous maturity. Increased syncytial knots are also associated with other abnormal conditions like preeclampsia (14).

The extraembryonic tissue such as the trophoblast gives rise to the placenta and provides the epithelial cover of the placental villous trees. They show high rates of proliferation and differentiation, as well as high rates of apoptosis. This villous trophoblast comes into direct contact with maternal blood displaying a continuous turnover of its layers. The villous trophoblast displays proliferation and differentiation of its precursor cells, villous cytotrophoblasts. They differentiate into multinucleated layer, the syncytiotrophoblast. A second differentiation stage takes place, with a final apoptotic shedding event, releasing apoptotic syncytial knots into the maternal circulation. As a normal process of trophoblast turnover apoptosis, the release of apoptotic material does not induce any inflammatory response of the mother $(15,16)$.

Pre-eclampsia is characterized by alteration in the balance between proliferation and apoptosis of villous trophoblast. This results in a dysregulated release of material from the syncytiotrophoblast into maternal blood. There is also an 
increasing release by necrosis. Therefore, due to ongoing apoptosis within the syncytiotrophoblast, the necrotic release of apoptotic material leads to aponecrotic shedding. Cell-free components of the syncytiotrophoblast like G-actin and DNA maybe able to damage the maternal endothelium and hence trigger pre-eclampsia. So, preeclampsia might be a result rather than a cause of this altered balance $(17,18)$.

Other previous studies claimed that degenerative changes of pre-eclamptic placentae may be related to an aberration in placentation. Placentation is a process of pseudovasculogenesis, where the trophoblasts invade the uteroplacental spiral arteries. The initially elastic and small caliber arteries are transformed into inelastic and dilated tubes with losing maternal vasomotor control. This modification provides adequate perfusion and oxygen supply for sustaining growth of fetus. This process is abnormal in women with preeclampsia. Where, the cytotrophoblasts fail to differentiate from epithelial phenotype to invasive endothelial phenotype. So, the uteroplacental spiral arteries remain only as small-caliber elastic vessels. This is failed, abnormal, or aberrant pseudovasculogenesis leads to reduced blood flow to the placenta, with subsequent placental ischemia or hypoxic placenta. A hypoxic placenta then releases many vasoactive factors such as tyrosine kinase-1, tumor necrosis factor $1-\alpha$, and interleukin-6. These factors results in decrease in nitric oxide bioavailability and an increase in reactive oxygen species $(5,9)$.

The present work found increased density of collagen fibers in the villous stroma and around fetal blood capillaries of PE group. This was confirmed by a significant increase in the mean area\% of collagen fibers. These results were in agreement with some authors $(19,20)$ who observed significant increase of collagen fibers in the villous stroma. This could be attributed to placental ischemia and increased levels of anti-angiogenic factor soluble fms-like tyrosine kinase-1 (sFlt-1). This consenquently may decrease uterine, placental and vascular matrix metalloproteinase-2 and -9, leading to increased uteroplacental and vascular collagen (21).

A significant decrease in immunoreactivity of eNOS (represented by area \%) was observed in PE group compared with the control group. This finding was inconsistant with previous studies $(22,23)$ that revealed similar immunolocalization of eNOS in syncytiotrphoblasts and endothelium of fetal capillaries in normal and preeclamptic placentae. Therefore, they excluded a possible pathogenic role of eNOS in this disease. On contrary, another study (24) demonstrated reduced eNOS expression in compromised pregnancies. Another study (25) demonstrated reduced fetal growth and fetoplacental blood flow at mid- and late gestation in eNOS knock out mice fetuses.

In spite of great debate about the role of eNOS in the pathogenesis of preeclampsia, nitric oxide (NO) produced by eNOS, is the main vasodilator in the placenta. It is involved in the regulation of feto-placental vascular reactivity, placental bed vascular resistance, trophoblast invasion, apoptosis, and platelet adhesion and aggregation in the intervillous space $(24,26,27)$. Plasma asymmetric dimethyl arginine (ADMA) is recognized as a biomarker of many disorders including endothelial disorders. Maternal plasma ADMA levels are reduced in a normal pregnancy. It increases in compromised pregnancies such as those with preeclampsia. This substance may decrease NO production by different ways such as reduced affinity of eNOS to the cofactor tetrahydrobiopterin (BH4) (28). Thus, manipulation of the ADMA-NO pathways may have a therapeutic potential to rescue placental blood flow and improve long-term outcomes in patients with placental insufficiencies.

In the current study, immunoexpression (represented by area \%) of HIF-1 $\alpha$ was significantly increased in PE group compared with the control group. This was in agreement with some authors (9) who demonstrated strong positive nuclear immuoreaction of HIF-1 $\alpha$ in sycytiotrophoblast cells of preeclamptic placentae.

Hypoxia-inducible factor- $1 \alpha(\mathrm{HIF}-1 \alpha)$ is a transcription factor that can not be detected unless cells are subjected to hypoxia. HIF-1 $\alpha$ is a key regulator for the induction of genes that facilitate adaptation and survival of cells from normoxia to hypoxia. During hypoxia, HIF-1 $\alpha$ becomes stabilized and translocates from the cytoplasm to the nucleus. It dimerizes with HIF-1 $\beta$ forming transcriptionally active complex. Then, it binds to hypoxia-response elements (HREs) in the promoters of several genes involved in the adaptation to an environment of hypoxia $(29,30)$.

However, HIF-1 $\alpha$ is known to activate the transcription factor of vascular endothelial growth factor (VEGF). So, it could promote hypoxic cells to survive under hypoxic conditions. This is because; VEGF is a key factor in angiogenesis (29). The increase in the levels of HIF-1 $\alpha$ was found to be closely related to the increase in the levels of another protein called telomerase. Telomerase plays an important role in cell growth and cell immortalization. In spite of this compensatory mechanism, HIF-1 $\alpha$ expression remains abnormally elevated under hypoxic conditions, and trophoblasts remain in a relatively immature state of differentiation in preeclamptic placenta. As a direct consequence, trophoblast invasion into the uterus is limited and uteroplacental perfusion is reduced. However, whether the increase in levels of this protein occurs in parallel with severe aberrant pseudovasculogenesis requires further investigation $(9,31)$.

The role of HIF-1 $\alpha$ in the pathogenesis of preeclampsia and whether it could be a marker of preeclampsia are still being investigated. However, it is suggested by some invistigators to measure the levels of this transcription factor more frequently to avoid a potential development of preeclampsia at a late trimester (8).

\section{Conclusion}

As evidenced by the previous results, this study concluded various placental changes detected by histological and immunohistochemical techniques. The findings have clarified a significant correlation between eNOS and HIF-1 $\alpha$ 
immunoexpression and pre-eclampsia. Whether this relation is causal or effect relationship, this may need further investigations for better management and avoidance of consequences of pre-eclampsia.

\section{References}

[1] Narasimha A, Vasudeva DS. Spectrum of changes in placenta in toxemia of pregnancy. Indian J Pathol Microbiol. 2011; 54(1):15-20.

[2] Akhlaq M, Nagi AH, Yousaf AW. Placental morphology in pre-eclampsia and eclampsia and the likely role of NK cells. Indian J Pathol Microbiol. 2012; 55(1):17-21.

[3] Reis ZSN, Lage EM, Teixeira PG, Porto LB, Guedes LR, De Oliveira ECL. Early onset pre-eclampsia: is it a better classification for maternal and perinatal outcomes?Rev Bras Ginecol Obstet. 2010; 32:584-90.

[4] Hutcheon JA, Lisonkova S, Joseph KS. Epidmiology of preeclampsia and the other hypertensive disorders of pregnancy. Best Pract Res Clin Obstet Gynaecol. 2011; 25:391-403.

[5] Powe CE, Levine RJ, Karumanchi SA. Preeclampsia, a disease of the maternal endothelium: the role of antiangiogenic factors and implications for later cardiovascular disease. Circulation. 2011; 123(24):2856-69.

[6] Lamarca B. Endothelial dysfunction. An important mediator in the pathophysiology of hypertension during pre-eclampsia. Minerva Ginecol. 2012; 64(4):309-20.

[7] Kulandavelu S, Whiteley KJ, Qu D, Mu J, Bainbridge SA, Adamson SL. Endothelial nitric oxide synthase deficiency reduces uterine blood flow, spiral artery elongation, and placental oxygenation in pregnant mice. Hypertension. 2012; 60(1):231-8.

[8] Akhilesh M, Mahalingam V, Nalliah S, Ali RM, Ganesalingam $\mathrm{M}$, Haleagrahara N. Hypoxia-inducible factor- $1 \alpha$ as a predictive marker in pre-eclampsia. Biomed Rep. 2013;1(2):257-8.

[9] Akhilesh M, Mahalingam V, Nalliah S, Ali RM, Ganesalingam M, Haleagrahara N. Participation of hypoxia-inducible factor$1 \alpha$ in the pathogenesis of preeclampsia-related placental ischemia and its potential as a marker for preeclampsia. Biomarkers and Genomic Med. 2014; 66(3):121-5.

[10] Kaufmann P, Huppertz B, Frank HG. The fibrinoids of the human placenta: origin, composition and functional relevance. Anat Anz. 1996; 178(6):485-501.

[11] Sedano-Balbas S, Lyons M, Cleary B, Murray M, Gaffney G, Maher M. Placental prothrombin mRNA levels in APC resistance (APCR) women with increased placental fibrin deposition. Ir J Med Sci. 2014; 183(3):477-80.

[12] Romero R, Whitten A, Korzeniewski SJ, Than NG, Chaemsaithong P, Miranda J, Dong Z, Hassan SS, Chaiworapongsa T. Maternal floor infarction/massive perivillous fibrin deposition: a manifestation of maternal antifetal rejection? Am J Reprod Immunol. 2013; 70(4):28598

[13] Stark MW, Clark L, Craver RD. Histologic differences in placentas of preeclamptic/eclamptic gestations by birthweight, placental weight, and time of onset. Pediatr Dev Pathol. 2014; 17(3):181-9.

[14] Loukeris K, Sela R, Baergen RN. Syncytial knots as a reflection of placental maturity: reference values for 20 to 40 weeks' gestational age. Pediatr Dev Pathol. 2010; 13(4):305-9.

[15] Huppertz B. Placental Villous Trophoblast: the Altered Balance Between Proliferation and Apoptosis Triggers Preeclampsia. J Rep Med Endocrin. 2006; 3 (2): 103-8.

[16] Huppertz B, Gauster M. Trophoblast fusion. Adv Exp Med Biol. 2011; 713:81-95.

[17] Gauster M, Moser G, Orendi K, Huppertz B. Factors involved in regulating trophoblast fusion: potential role in the development of preeclampsia. Placenta. 2009; 30 Suppl A: S49-54.

[18] Dechend R, Staff AC. Placenta Messages to the Mother: not just debris. Hypertension. 2012; 59: 191-3.

[19] Atwa KA, Farghaly LM. Light and electron microscopic study of placental tissue in women suffering from severe preeclampsia. E J H. 2011; 34(4): 650-61.

[20] Shehab AA. The human placenta in pre-eclampsia: histological, immunohistochemical and ultrasonographic study. E J A. 2012; 35(3):105-20.

[21] Li W, Mata KM, Mazzuca MQ, Khalil RA. Altered matrix metalloproteinase-2 and -9 expression/activity links placental ischemia and anti-angiogenic sFlt-1 to uteroplacental and vascular remodeling and collagen deposition in hypertensive pregnancy. Biochem Pharmacol. 2014; 89(3):370-85.

[22] Orange SJ, Painter D, Horvath J, Yu B, Trent R, Hennessy A. Placental endothelial nitric oxide synthase localization and expression in normal human pregnancy and pre-eclampsia. Clin Exp Pharmacol Physiol. 2003; 30(5-6):376-81.

[23] Corthorn J, Germain AA, Chacón C, Rey S, Soto GX, Figueroa CD, Müller-Esterl W, Duarte I, Valdés G. Expression of kallikrein, bradykinin b2 receptor, and endothelial nitric oxide synthase in placenta in normal gestation, preeclampsia, and placenta accreta. Endocrine. 2006; 29(3):491-9.

[24] Huang LT, Hsieh CS, Chang KA, Tain YL. Roles of nitric oxide and asymmetric dimethylarginine in pregnancy and fetal programming. Int J Mol Sci. 2012; 13(11):14606-22.

[25] Kulandavelu S, Whiteley KJ, Bainbridge SA, Qu D, Adamson SL. Endothelial NO synthase augments fetoplacental blood flow, placental vascularization, and fetal growth in mice. Hypertension. 2013; 61(1):259-66.

[26] Reynolds LP, Borowicz PP, Caton JS, Vonnahme KA, Luther JS, Buchanan DS, Hafez SA, Grazul-Bilska AT, Redmer DA. Uteroplacental vascular development and placental function: an update. Int J Dev Biol. 2010; 54: 355-66.

[27] Krause BJ, Hanson MA, Casanello P. Role of nitric oxide in placental vascular development and function. Placenta. 2011; 32:797-805.

[28] Zoltan K, Sandor V. Nitric oxide and preeclampsia. Orv. Hetil. 2010; 151: 2125-35.

[29] Zhu H, Bunn FH. How Do Cells Sense Oxygen? Science. 2001; 292(5516): 449-51. 
[30] Trendeleva TA, Aliverdieva DA, Zvyagilskaya RA. Mechanisms of sensing and adaptive responses to low oxygen conditions in mammals and yeasts. Biochemistry (Mosc). 2014; 79(8):750-60.
[31] Nishi H, Nakada T, Kyo S, Inoue M, Shay JW, Isaka K. Hypoxia-inducible factor 1 mediates upregulation of telomerase (hTERT). Mol Cell Biol. 2004; 24(13):6076-83. 